\title{
Serum/glucocorticoid-induced protein kinase-1 facilitates androgen receptor-dependent cell survival
}

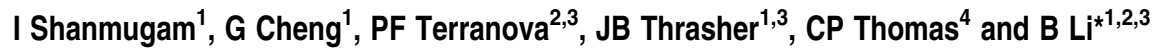

Androgen receptor (AR) is a critical factor in the development and progression of prostate cancer. We and others recently demonstrated that eliminating AR expression leads to apoptotic cell death in AR-positive prostate cancer cells. To understand the mechanisms of AR-dependent survival, we performed a genome-wide search for AR-regulated survival genes. We found that serum/glucocorticoid-induced protein kinase-1 (SGK-1) mRNA levels were significantly upregulated after androgen stimulation, which was confirmed to be AR dependent. Promoter analysis revealed that the AR interacted with the proximal and distal regions of the sgk1 promoter, leading to sgk-1 promoter activation after androgen stimulation. Functional assays demonstrated that SGK-1 was indispensable for the protective effect of androgens on cell death induced by serum starvation. SGK-1 overexpression not only rescued cells from AR small-interfering RNA (siRNA)-induced apoptosis, but also enhanced AR transactivation, even in the absence of androgen. Additionally, SGK-1 siRNA reduced AR transactivation, indicating a positive feedback effect of SGK-1 expression on AR-mediated gene expression and cellular survival. Taken together, our data suggest that SGK-1 is an androgen-regulated gene that plays a pivotal role in AR-dependent survival and gene expression.

Cell Death and Differentiation (2007) 14, 2085-2094; doi:10.1038/sj.cdd.4402227; published online 12 October 2007

Androgens play a critical role not only in the physiological development of the prostate but also in the genesis of prostate cancer (reviewed by Heinlein and Chang ${ }^{1}$ ). These hormones regulate the activity of the androgen receptor $(A R)$, a ligandactivated transcription factor and a member of the nuclear receptor superfamily. ${ }^{2}$ Hormone-receptor complexes bind to certain hormone responsive DNA sequences proximal to target genes and regulate their expression. ${ }^{1-3}$

It is well demonstrated that castration in rodent models or androgen ablation in cell culture system causes a rapid apoptotic response in the prostate-derived epithelial cells. ${ }^{4,5}$ Based on these phenomena, androgen ablation therapy has been used in clinical management of prostate cancer patients. ${ }^{6}$ However, the disease progresses to a more aggressive androgen-independent stage (termed 'hormonerefractory' because the tumor is resistant to androgen ablation therapy). ${ }^{7}$ The etiology of hormone-refractory progression may have various molecular causes, but a critical role of the AR has emerged..$^{8-11}$ We and others have recently showed that eliminating $A R$ expression led to a profound apoptotic response in AR-positive prostate cancer cells, suggesting that the AR is essential for cellular survival in prostate cancers. ${ }^{12-15}$ However, the downstream effectors that facilitate AR-dependent survival are not fully elucidated.
Serum and glucocorticoid-induced protein kinase-1 (SGK1) belongs to the 'AGC' subfamily of protein kinases, including protein kinase $A, G$, and $C .^{16}$ There are two more isoforms of SGK-1 in mammalian cells, namely SGK-2 and -3 . They are evolutionally conserved and share an $80 \%$ homology in the catalytic domains. ${ }^{17}$ Particularly, SGK-1 shares about $54 \%$ identity of its catalytic domain with a major cellular survival factor protein kinase B (PKB, also called Akt), indicating a functional similarity. In fact, like Akt, SGK-1 is activated by phosphorylation through a phosphatidylinositol 3-kinase (PI3K)-dependent signal pathway. ${ }^{18,19}$ However, unlike other protein kinases in AGC family, such as Akt, SGK-1 but not SGK-2 or -3 is regulated and activated mainly through a transcriptional mechanism by extracellular stimuli. Its promoter contains several consensus sequences for transcription factors that can be activated by a very large spectrum of stimuli besides glucocorticoid and serum. ${ }^{19,20}$ Upon induction, it acts as a cell survival factor in different situations and is also able to regulate numerous membrane transporters and channel proteins. ${ }^{18,19}$

In the studies reported here we identified a downstream effecter pathway responsible for AR-dependent survival. We found that expression of SGK-1 but not other isoforms or Akt was upregulated by androgen stimulation. Further analysis confirmed that SGK-1 is an androgen-induced gene via an

\footnotetext{
${ }^{1}$ Department of Urology, The University of Kansas Medical Center, Kansas City, KS 66160, USA; ${ }^{2}$ Department of Molecular and Integrative Physiology, The University of Kansas Medical Center, Kansas City, KS 66160, USA; ${ }^{3}$ Kansas Masonic Cancer Research Institute, The University of Kansas Medical Center, Kansas City, KS 66160, USA and ${ }^{4}$ Department of Internal Medicine, University of lowa College of Medicine, lowa City, IA 52246, lowa

${ }^{*}$ Corresponding author: B Li, Department of Urology, KUMC Urology, 3901 Rainbow Blvd, Lied 1042, Kansas City, KS 66160, USA. Tel: +1913 588 4773;

Fax: + 1913588 7625; E-mail: bli@kumc.edu

Keywords: SGK-1; androgen receptor; prostate cancer; survival; gene regulation

Abbreviations: AAV, adeno-associated virus; AR, androgen receptor; ARHP, AR hairpin; cDNA, complementary DNA; ChIP, chromatin immunoprecipitation; cFBS, charcoal-stripped FBS; CMV, cytomegalovirus; CSDX, Casodex; FBS, fetal bovine serum; GRE, glucocorticoid response element; I $\mathrm{B}$ - $\alpha$, I kappa B-alpha; IKK $\beta$, I $\kappa \mathrm{B}$ kinase $\beta$; NF- $\kappa \mathrm{B}$, nuclear factor- $\kappa \mathrm{B}$; PB, probasin; PBS, phosphate-buffered saline; PCR, polymerase chain reaction; PI3K, phosphoinositide 3-kinases; PKB, protein kinase B; PSA, prostate-specific antigen; RT, reverse transcription; SE, standard error; SEAP, secreted alkaline phosphatase; SGK-1, serum/glucocorticoid-induced protein kinase-1; siRNA, small-interfering RNA; SRE, steroid response element; TBS-T, Tris-buffered saline/Tween-20

Received 20.2.07; revised 20.8.07; accepted 28.8.07; Edited by JA Cidlowski; published online 12.10.07
} 
AR-dependent transcriptional mechanism. Functional assays demonstrated that SGK-1 is indispensable from AR-mediated survival. SGK-1 overexpression enhances AR-mediated gene expression and reduces cell death induced by $A R$ small interfering RNA (siRNA), suggesting a positive feedback effect of androgen-induced SGK-1 expression on AR-dependent gene expression and survival.

\section{Results}

AR-dependent SGK-1 expression after androgen stimulation. We and others recently demonstrated that the AR is essential for cellular survival of AR-positive prostate cancer cells. ${ }^{12-15}$ To better understand the mechanism of AR-dependent survival, we performed a genome-wide search for AR-regulated survival genes using complementary DNA (cDNA) microarray analysis. Prostate cancer LNCaP cells were serum-starved for $48 \mathrm{~h}$, and were then treated with the solvent or a synthetic androgen R1881 $(1.0 \mathrm{nM})$ for $8 \mathrm{~h}$. The Affymetrix human genome U133A chips were used to analyze the alterations of androgen-induced gene expression. SGK-1 was identified as one of the genes significantly upregulated by androgen stimulation compared to the solvent control (Table 1). Other SGK isoforms and the closest relative protein kinases Akt-1 or -3 remained unchanged after androgen stimulation. These data were supported by a recent publication from another group using the same strategy, which showed an 8.1-fold increase of SGK-1 expression by androgen treatment. ${ }^{21}$

We confirmed the microarray data using additional methods, including conventional reverse transcription (RT)polymerase chain reaction (PCR) and real-time RT-PCR assays for mRNA expression, as well as western blot for protein expression. LNCaP cells were serum-starved for $48 \mathrm{~h}$ and then treated with R1881 for $8 \mathrm{~h}$ (mRNA analysis) or $24 \mathrm{~h}$ (protein analysis) under serum-free conditions. To test for AR dependency, the AR antagonist bicalutamide (Casodex ${ }^{\mathrm{TM}}, \mathrm{CSDX}$ ) were used to pre-treat the cells before R1881 addition. A well-known AR-dependent gene prostatespecific antigen ( $P S A)$ was included in the analysis as a positive control. Ribosomal 28S RNA was used as a loading control for RT-PCR experiments. As illustrated in Figure 1a, SGK-1 expression was not detectable under the conditions of serum starvation. Similar to $P S A$, SGK-1 expression was dramatically increased by R1881 stimulation. The R1881 effect was blocked by CSDX pretreatment, indicating that androgen-induced SGK-1 expression is AR dependent. AR-mediated SGK-1 expression was further confirmed using real-time RT-PCR (Figure 1b) and Western blot (Figure 1c). However, there was no significant alteration of Akt-1 expression after R1881 treatment, consistent with the data from cDNA microarray analysis (Table 1). These results suggest that SGK-1 is an androgen-inducible gene via AR-dependent transcription in prostate cancer cells.

As mentioned earlier, AR gene silencing results in apoptotic cell death. ${ }^{12-15}$ Since the survival kinase SGK-1 is an
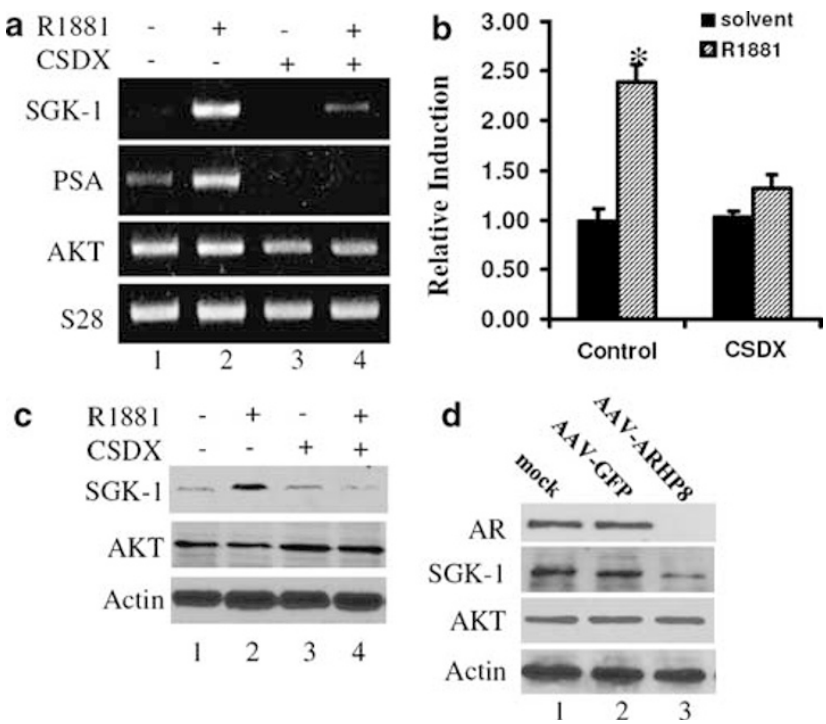

Figure 1 Androgen receptor regulates SGK-1 expression. (a, b) LNCaP cells were serum-starved for $48 \mathrm{~h}$ and then pretreated with the anti-androgen bicalutamide (CSDX) at $10 \mu \mathrm{M}$ or the solvent for $30 \mathrm{~min}$. Cells were then stimulated with the synthetic androgen R1881 at $1.0 \mathrm{nM}$ in serum-free media for $8 \mathrm{~h}$ before harvest. Total RNA was extracted by TriZo ${ }^{\mathrm{TM}}{ }^{-}$-based protocol. Gene expression at mRNA level was assessed by RT-PCR (a) or real-time RT-PCR (b). For RT-PCR, human psa gene served as positive control of androgen action while $28 \mathrm{~S}$ ribosomal gene was used as negative control. A set of the representative data was shown. For the real-time RT-PCR, the expression level of $s g k-1$ gene was normalized against $\beta$-actin expression level before the relative value was calculated compared to the solvent control (set as value 1.0). Data represent three separate experiments. Error bar represents the standard error (S.E.), and the asterisk indicates a significant difference $(P<0.05$, $t$-test) between R1881 treatment and the solvent control. (c) After bicalutamide pretreatment as above, cells were treated with R1881 (1.0 nM) for $24 \mathrm{~h}$ and were then harvested. Whole cellular protein extracts were used for Western blot to assess protein expression levels of SGK-1 and Akt. Membranes were reprobed with anti-Actin as the loading control. (d) LNCaP cells were infected with AAV-GFP or AAV-ARHP8 at $1.0 \times 10^{3}$ viral particles per cell in serum-reduced media ( $2 \%$ cFBS) for 5 days. A mock infection was included as negative control. Whole cell lysates were used for Western blot to determine protein levels of AR, SGK-1, and Akt. Anti-Actin blot served as a loading control. Data represent two separate experiments

Table 1 cDNA microarray analysis of androgen-stimulated gene expression

\begin{tabular}{|c|c|c|c|c|}
\hline Gene symbol & Probe identification & Fold induction & Control signal & Treatment signal \\
\hline $\begin{array}{l}\text { SGK-1 } \\
\text { SGK-2 } \\
\text { SGK-3 } \\
\text { AKT-1 } \\
\text { AKT-3 } \\
\text { GAPDH }\end{array}$ & $\begin{array}{l}201739 \text { _at } \\
213837 \text { _at } \\
220038 \text { _at } \\
207163 \text { _s_at } \\
219393 \text { _s_at } \\
212581 \text { _x_at }\end{array}$ & $\begin{array}{l}6.62 \\
1.09 \\
1.72 \\
0.79 \\
1.82 \\
1.01\end{array}$ & $\begin{array}{c}71.9 \\
139.2 \\
173.8 \\
1,496 \\
96.1 \\
25327\end{array}$ & $\begin{array}{c}475.8 \\
152 \\
298.5 \\
1192 \\
175.1 \\
25767\end{array}$ \\
\hline
\end{tabular}


AR-regulated gene, we asked if AR silencing led to SGK-1 downregulation, which consequently led to apoptotic cell death. To test this hypothesis, we measured SGK-1 protein expression after AR gene silencing. To introduce the AR siRNA more efficiently, we created an adeno-associated virus (AAV) that carries a hairpin-structured AR siRNA (AAV-ARHP8) expression cassette based on the AR siRNA \#8 sequence used in our previous work. ${ }^{12}$ LNCaP cells were infected with the AAV-ARHP8 viruses or a control empty virus (AAV-GFP) for 5 days in androgen withdrawal media ( $2 \%$ charcoal-stripped fetal bovine serum (FBS), cFBS). As shown in Figure 1d, AAV-ARHP8 infection resulted in a complete loss of AR expression. In parallel, the protein level of SGK-1 but not Akt was reduced dramatically in AAV-ARHP8infected cells compared to the control cells. These results confirmed our hypothesis that SGK-1 expression is regulated by the AR through either androgen-dependent or -independent mechanisms, such as growth factors and cytokines. ${ }^{7}$ The detailed pathways are under further investigation by our group.
AR activates SGK-1 promoter following androgen stimulation. The AR, together with the receptors of glucocorticoid, progesterone and mineralocorticoid, are closely related members (class I) in the nuclear receptor superfamily. ${ }^{22}$ They all recognize similar response elements (steroid response element, SRE), which are organized as inverted repeats of $5^{\prime}$-TGTTCT-3'-like sequences with a threenucleotide spacer. ${ }^{23} \mathrm{We}$ and others have shown that SGK1 expression is regulated by glucocorticoid via an imperfect glucocorticoid response element (GRE) on the sgk1 promoter. ${ }^{16,24,25}$ Therefore, we tested if androgen activates sgk1 promoter. We used a luciferase reporter (F4R4-Luc) that was described in our previous publication. ${ }^{24}$ This reporter uses a $-3142 /+117$ fragment relative to the transcription starting site of human sgk1 gene as the promoter (Figure 2a). LNCaP (mutant AR) and LAPC-4 (wild-type AR) cells were transfected with the F4R4-Luc and the control reporter constructs. Following serum starvation for $24 \mathrm{~h}$ and bicalutamide pretreatment for $45 \mathrm{~min}$, cells were stimulated with R1881 for $24 \mathrm{~h}$ in androgen withdrawal media
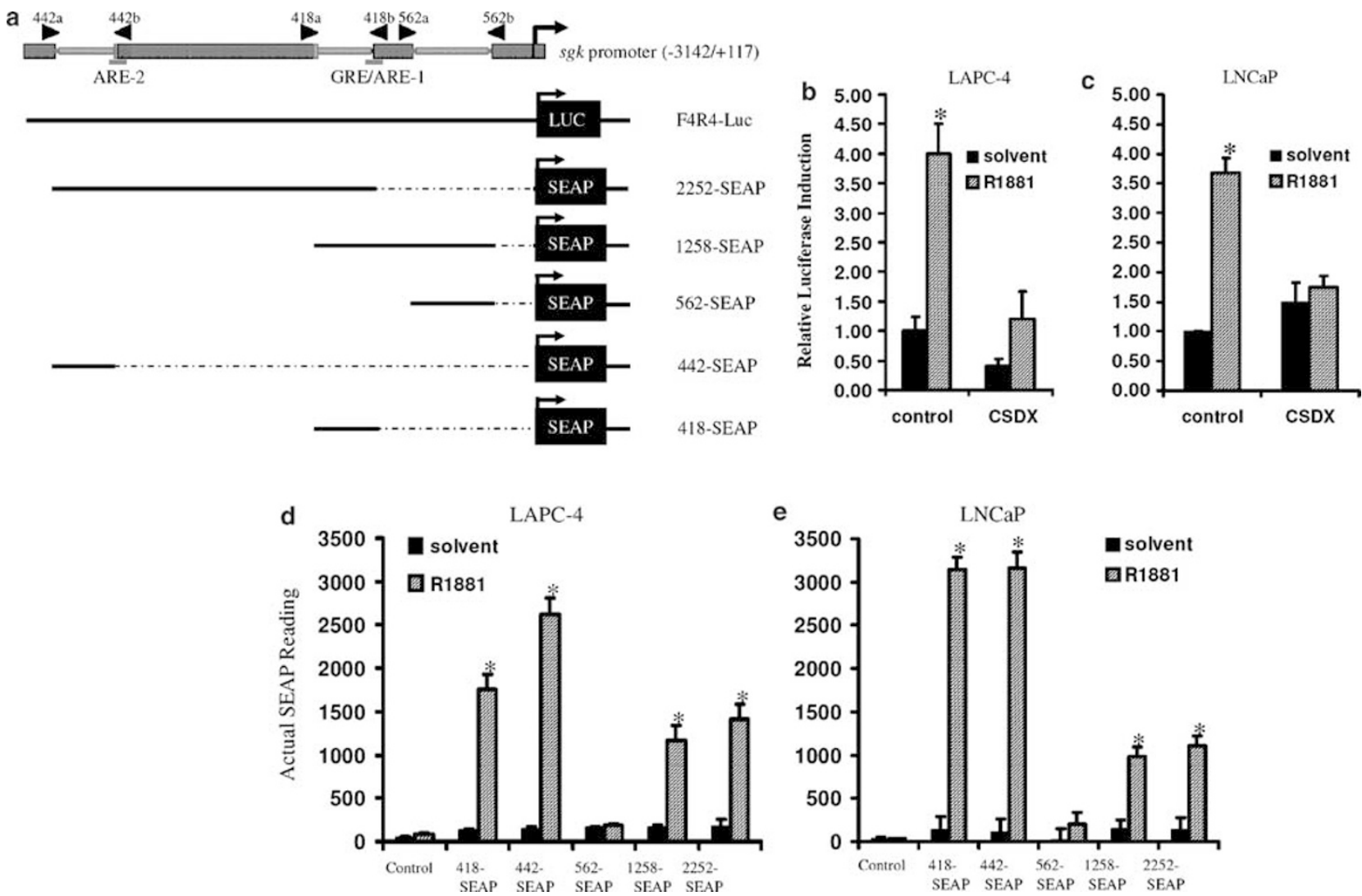

Figure 2 Androgen receptor transactivates sgk-1 promoter. (a) Schematic illustration of $s g k-1$ promoter, the GRE/ARE-1 and the putative secondary ARE-2 location, ChIP primer binding sites, and structure of SEAP gene reporters driven by different fragments of sgk-1 promoter. (b, c) LAPC-4 or LNCaP cells were transfected with sgk-1 promoter-driven luciferase reporter (pGL3-SGK1/F4R4-LUC) together with an internal control reporter pCMV-SEAP. After serum starvation, cells were pretreated with bicalutamide (CSDX, $10 \mu \mathrm{M}$ ) or the solvent, and were then stimulated with R1881 $(1.0 \mathrm{nM})$ for $24 \mathrm{~h}$ in $5 \%$ cFBS-containing media. Culture supernatants were collected for SEAP assay and cellular proteins were used for luciferase assay. Luciferase activities were normalized against the corresponding protein concentration and SEAP activity before the relative fold induction was calculated compared to the solvent control. (d, e) Cells as indicated were transfected with different SEAP reporter constructs, and a mock transfection was included with transfection reagent only. Following serum starvation, cells were stimulated with R1881 (1.0 nM) or the solvent for $24 \mathrm{~h}$ in $5 \% \mathrm{cFBS}$-containing media. Culture supernatants were collected for SEAP assay and cells were harvested for protein assay. SEAP activities were normalized against their corresponding cellular protein levels. Data represent three different experiments, and the error bar indicates S.E. The asterisk indicates a significant difference $(P<0.05, t$-test) between R1881 treatment and the solvent control 
(5\% cFBS). As shown in Figure $2 b, \mathrm{R} 1881$ significantly increased the reporter activity in both cell lines, and bicalutamide pretreatment abolished R1881-stimulated F4R4-LUC activation. These data confirmed that androgen activates SGK-1 promoter via the AR.

To narrow down the position of $A R$ responsive elements within the $s g k 1$ gene promoter, we generated five different secreted alkaline phosphatase (SEAP) reporter constructs using different fragments from the sgk1 gene promoter. These fragments were PCR-amplified from LNCaP cell genomic DNA, as illustrated in Figure 2a. The proximal promoter $(-1548 /-290)$ was termed as 1258-SEAP, which contains the GRE motif $(-1147 /-1133)$ identified previously. Using this proximal promoter, we constructed two truncated promoters, named as 418-SEAP $(-1548 /-1130$ with the GRE motif) and 562-SEAP (-852/-290 without the GRE motif). The distal promoter 2252-SEAP $(-3382 /-1130)$ and its truncated one 442-SEAP $(-3382 /-2940)$ contain several putative SRE motifs, as suggested by a computer-based inspection. ${ }^{19}$ The androgen responsiveness of these reporters was analyzed in LNCaP and LAPC-4 cells. As shown in Figure $2 d$ and e, R1881 treatment significantly activated the reporters driven by the proximal (1258-SEAP) and distal (2252-SEAP) promoter fragments in both cell lines. This responsiveness was further increased in those truncated fragments of the proximal (418-SEAP) and the distal (442-SEAP) promoters. This increased response to androgen stimulation from the truncated distal promoter (442-SEAP) compared to the distal (2252-SEAP) promoter indicated the existence of a potential secondary ARE motif (ARE-2) within the far distal region of the sgk1 gene promoter, as illustrated in Figure 2a. In contrast, the truncated minimum promoter (562-SEAP) lacking the GRE motif did not response to R1881 stimulation, thereby confirming that the proximal GRE (hereafter termed as ARE1 ) is required for androgen-induced SGK-1 expression.

Then, we went on to determine if the AR physically interacts with the ARE-containing regions on the sgk1 gene promoter we had indicated using the reporter assays. Serum-starved LNCaP cells were pretreated with CSDX followed by R1881 stimulation. Chromatin immunoprecipitation (ChIP) assays were used to analyze the AR interaction with genomic DNA in vivo. We chose to test three different regions on the sgk1 promoter: (a) the close proximal region, (b) the GRE/ARE-1 region, and (c) the far distal region, as illustrated in Figure 2a. Without R1881 stimulation, no interaction was detected between the AR and any promoter regions (Figure 3, lane 1). R1881 stimulation led to a clear signal of the AR interaction with the regions that contain either the GRE/ARE-1 motif or the ARE-2 motif in the far distal fragment. Consistent with the data from 562-SEAP reporter assay that showed no androgen responsiveness, no interaction was detected between the $\mathrm{AR}$ and the proximal promoter region. These results were in agreement with the conclusion from the reporter gene assays, and show that the AR regulates SGK-1 expression by binding to two sites in the promoter region of the sgk-1 gene. The secondary ARE-2 motif resides in the far distal region of the sgk1 gene promoter, as proposed by others previously. ${ }^{19}$

SGK-1 is required for AR-dependent survival. Similar to Akt, SGK-1 has been shown to be a cellular survival

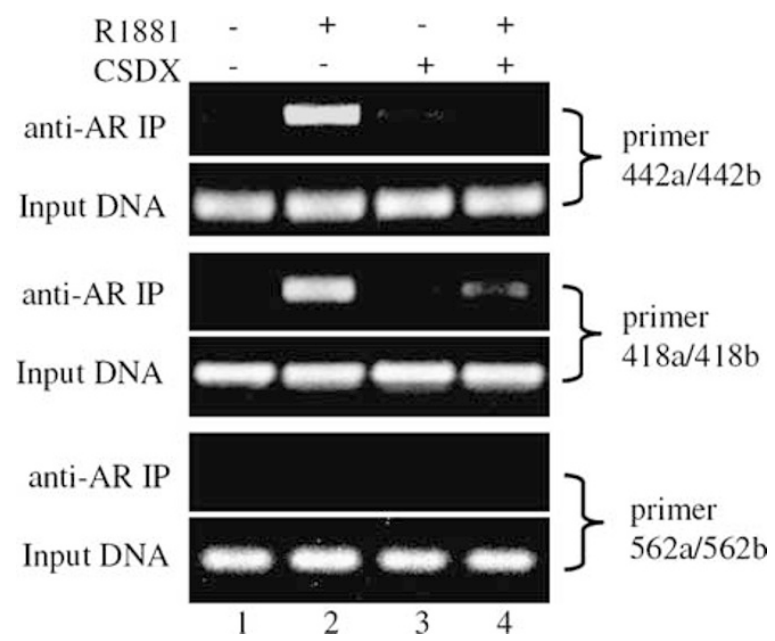

Figure 3 Androgen receptor interacts with the ARE locus on the sgk1 promoter. $\mathrm{LNCaP}$ cells were serum-starved for 2 days and were then pretreated with bicalutamide (CSDX, $10 \mu \mathrm{M})$. After stimulation with R1881 (1.0nM) or the solvent in $2 \%$ cFBS-containing media, cells were subjected to ChIP assay, as described. ${ }^{38}$ Anti-AR immunoprecipitated chromatin DNA were amplified with three different primer sets, and $10 \%$ of whole cellular genomic DNA were used as PCR templates as the positive control. Data represent two different experiments

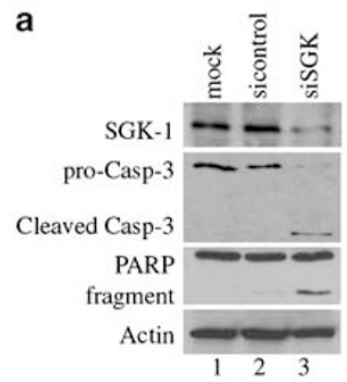

b clonogenic survival

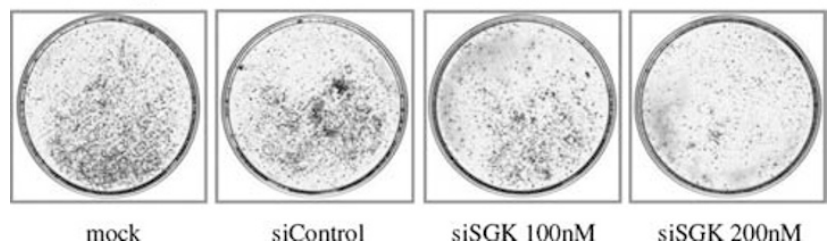

Figure 4 SGK-1 siRNA induces apoptotic cell death. (a) LAPC-4 cells were transfected with SGK-1 siRNA or the negative control siRNA at a final concentration of $100 \mathrm{nM}$ in media. A mock transfection was included as a negative control. Cells were cultured in androgen withdrawal media ( $2 \%$ cFBS) for 5 days. Whole cell lysates were then used for western blot with antibodies as indicated. Actin blot served as the loading control. Data represent three separate experiments. (b) LNCaP cells were transfected with the negative siRNA or SGK-1 siRNA at different concentrations as indicated in androgen withdrawal media ( $2 \% \mathrm{cFBS})$. After 7 days, survived colonies were stained and photographed. Data represent two separate experiments

factor. ${ }^{26-29}$ Therefore, we examined the role of SGK-1 in AR-dependent survival of prostate cancer cells. First, we tested if eliminating SGK-1 expression induces apoptosis. LAPC-4 cells were transfected with SGK-1 siRNA or a control siRNA, and were then kept in androgen withdrawal media 
(2\% cFBS) for 5 days. As shown in Figure 4a, transfection with the SGK-1 siRNA but not the control siRNA resulted in a dramatic loss of SGK-1 expression. In parallel, cell death was observed (cell round-up and floating in media under inverted microscope, data not shown) in cells transfected with SGK-1 siRNA but not with the control siRNA. After cell harvesting, we examined two apoptotic hallmarks, caspase-3 activation and PARP cleavage, to determine if SGK-1 siRNA-induced cell death was an apoptotic response. As expected, caspase3 activation and PARP cleavage were observed in SGK-1 siRNA-transfected cells but not in the controls (Figure 4a). These data suggest that SGK-1 elimination leads to apoptosis, which is supported by previous reports. ${ }^{26-29}$

Next, we confirmed that SGK-1 siRNA could induce apoptosis in another prostate cancer cell line. LNCaP cells were tested using a clonogenic assay. After transfection with the control or SGK-1 siRNA, cells were cultured in the same condition ( $2 \%$ cFBS) for 7 days. Surviving cells were visualized by crystal violet staining. As shown in Figure $4 \mathrm{~b}$, the surviving cell fraction was remarkably reduced in cells transfected with SGK-1 siRNA, especially in cells transfected with higher dose of SGK-1 siRNA, compared to the control siRNA-transfected cells. These data confirmed that SGK-1 is important for prostate cancer cell survival.

Since androgen was found to regulate SGK-1 expression, we asked if SGK-1 is required for androgen-induced survival in prostate cancer cells. LAPC-4 cells were transfected with SGK-1 siRNA or the control siRNA and cell death was initiated by serum starvation in the presence or absence of R1881. As shown in Figure 5a, in mock and control siRNAtransfected cells, serum starvation resulted in a moderate level of cell death. The death response was dramatically increased in cells transfected with SGK-1 siRNA. As expected, R1881 addition significantly reduced serum starvation-induced cell death in mock and control siRNA-transfected cells but not in SGK-1 siRNA-transfected cells. These results indicate that SGK-1 is important in androgen-induced survival.

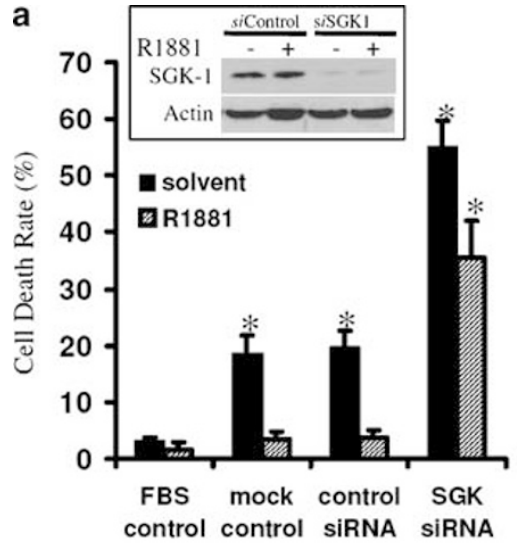

C

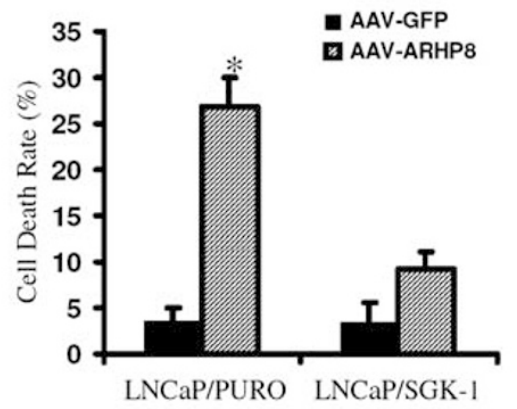

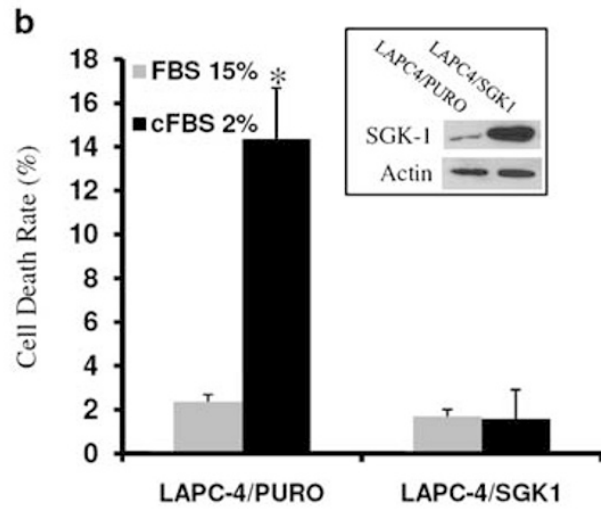

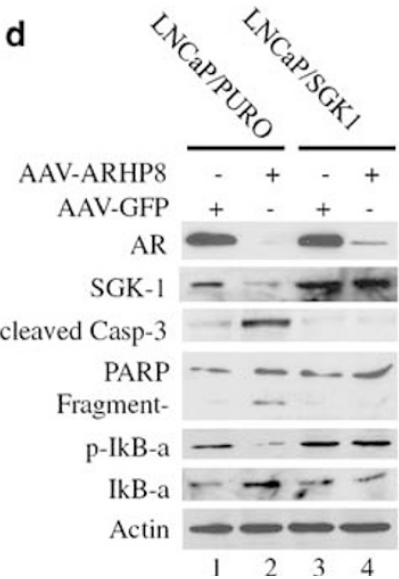

Figure 5 SGK-1 facilitates androgen receptor-dependent survival. (a) LAPC-4 cells were transfected with the control siRNA or SGK-1 siRNA (100 nM), and a mock transfection was also included. Transfected cells were cultured in serum-free media, and the untransfected control cells were cultured in a full culture media with serum (15\% FBS) in the presence or absence of R1881 $(1.0 \mathrm{nM})$ for 5 days. Cell death rates were assessed with trypan blue exclusion assay. The inserted immunoblotting panels show the efficacy of SGK-1 siRNA and the antibodies as indicated on the left-hand side. (b) LAPC-4 stable sublines LAPC-4/SGK1 and LAPC-4/PURO were cultured in either full growth media (15\% FBS) or androgen-withdrawal media ( $2 \%$ cFBS) for 10 days. Cell death rates were determined as above (a) by trypan blue exclusion assay. The inserted immunoblotting panels show the level of SGK-1 overexpression in the sublines, and the antibodies as indicated on the left-hand side. (c) LNCaP stable sublines LNCaP/SGK1 and LNCaP/PURO were infected with the AAVs as indicated at $1.0 \times 10^{3}$ viral particles per cell in serum-free media for 7 days. Cell death rates were determined by trypan blue exclusion assay $(\mathbf{a}, \mathbf{b})$ and cellular proteins were used for western blot analysis with the antibodies as indicated on the left-hand side (d). Data represent three separate experiments. For panel $\mathbf{a}, \mathbf{b}$, and $\mathbf{c}$, error bar indicates S.E. and the asterisk indicates a significant difference $(P<0.05, t$-test) compared to FBS control (a, $\mathbf{b})$ or AAV-GFP infected cells (c) 
Subsequently, we tested to determine if overexpression of SGK-1 would protect cells from cell death induced by androgen withdrawal. An LAPC-4 subline was established that overexpresses SGK-1 (LAPC-4/SGK1). A companion control line (LAPC-4/PURO) harbors an empty vector. Cells were kept in complete culture media (15\% FBS) or in androgen-withdrawal condition ( $2 \% \mathrm{cFBS})$. Cell death was determined at day 10. As shown in Figure $5 \mathrm{~b}$, androgen withdrawal induced a significant cell death in the control LAPC-4/PURO cells but not in LAPC-4/SGK1 cells, indicating again that SGK-1 is a potent survival factor in protecting against cell death after androgen withdrawal.

We showed above (Figure 1d) that AR silencing led to downregulation of SGK-1 in LNCaP cells. To understand the role of SGK-1 in AR-dependent survival, we tested whether SGK-1 overexpression could rescue cells from AR siRNAinduced cell death. ${ }^{12,13}$ An LNCaP subline was established that stably overexpresses SGK-1 (LNCaP/SGK1), and its control subline was generated with an empty vector (LNCaP/ PURO). Cell death was monitored after infecting the cells with AAV-ARHP8 virus under serum-free conditions. The empty virus AAV-GFP served as the control. The cell death rate was assessed 7 days later. As expected, AAV-ARHP8 virus induced a significant cell death compared to the control virus in the control LNCaP/PURO cells (Figure 5c), a finding consistent with our previous publication. ${ }^{12}$ However, AAVARHP8 virus-induced cell death was significantly reduced in LNCaP/SGK1 cells. Caspase-3 activation and PARP cleavage were also largely suppressed in LNCaP/SGK1 cells compared to the LNCaP/PURO control cells (Figure 5d). Together with the data shown in Figure 1d, these results indicate that SGK-1 plays a pivotal role in AR-dependent survival.
Recently, SGK-1 has been found to promote cellular survival by activating $\mathrm{l} \kappa$-B kinase $\beta(\mathrm{IKK}-\beta)$. This effect leads to increased I kappa B-alpha $\left(I_{\kappa} \mathrm{B}-\alpha\right)$ phosphorylation and nuclear factor- $\kappa \mathrm{B}$ (NF- $\kappa \mathrm{B})$-dependent survival, ${ }^{28}$ and also suppresses FOXO3 transactivation. ${ }^{29}$ Therefore, we tested to see if $\mathrm{I} \kappa \mathrm{B}-\alpha$ phosphorylation was altered after $\mathrm{AR}$ silencing or SGK-1 overexpression. As shown in Figure $5 d$, $1 \kappa \mathrm{B}-\alpha$ phosphorylation was largely reduced in LNCaP/PURO cells after AR silencing compared to the control (lane 2 versus lane 1), which was associated with SGK-1 downregulation. In contrast, higher levels of $\mathrm{I}_{\kappa} \mathrm{B}-\alpha$ phosphorylation were observed in LNCaP/SGK1 cells compared to the control LNCaP/ PURO cells, even after AR silencing (lanes 3 and 4). On the other hand, the total level of $I_{\kappa} \mathrm{B}-\alpha$ protein was increased in LNCaP/Puro cells after AR silencing, reflecting a reduced $I_{\kappa} \mathrm{B}-\alpha$ degradation due to a lack of phosphorylation. ${ }^{30}$ These data suggest that NF- $\kappa$ B activation after SGK-1-induced $I_{\kappa} \mathrm{B}-\alpha$ phosphorylation and degradation might be a part of the mechanisms involved in AR-dependent SGK-1-mediated survival in prostate cancer cells. ${ }^{31}$

SGK-1 modulates AR-mediated gene expression. Similar to the regulation of Akt, sgk-1 also can be activated via a PI3K-dependent mechanism. ${ }^{32}$ We previously showed that PI3K activity (but not Akt) is required for AR-mediated gene expression. ${ }^{33}$ Therefore, we determined whether SGK-1 modulates AR transactivation. First, we tested whether SGK-1 overexpression could enhance androgen-stimulated AR transactivation. LNCaP and LAPC-4 cells were transfected with an $A R$ responsive gene reporter (ARELUC) together with increasing amounts of constructs expressing SGK-1. After serum starvation, cells were treated with R1881 or the solvent for $24 \mathrm{~h}$. As shown in
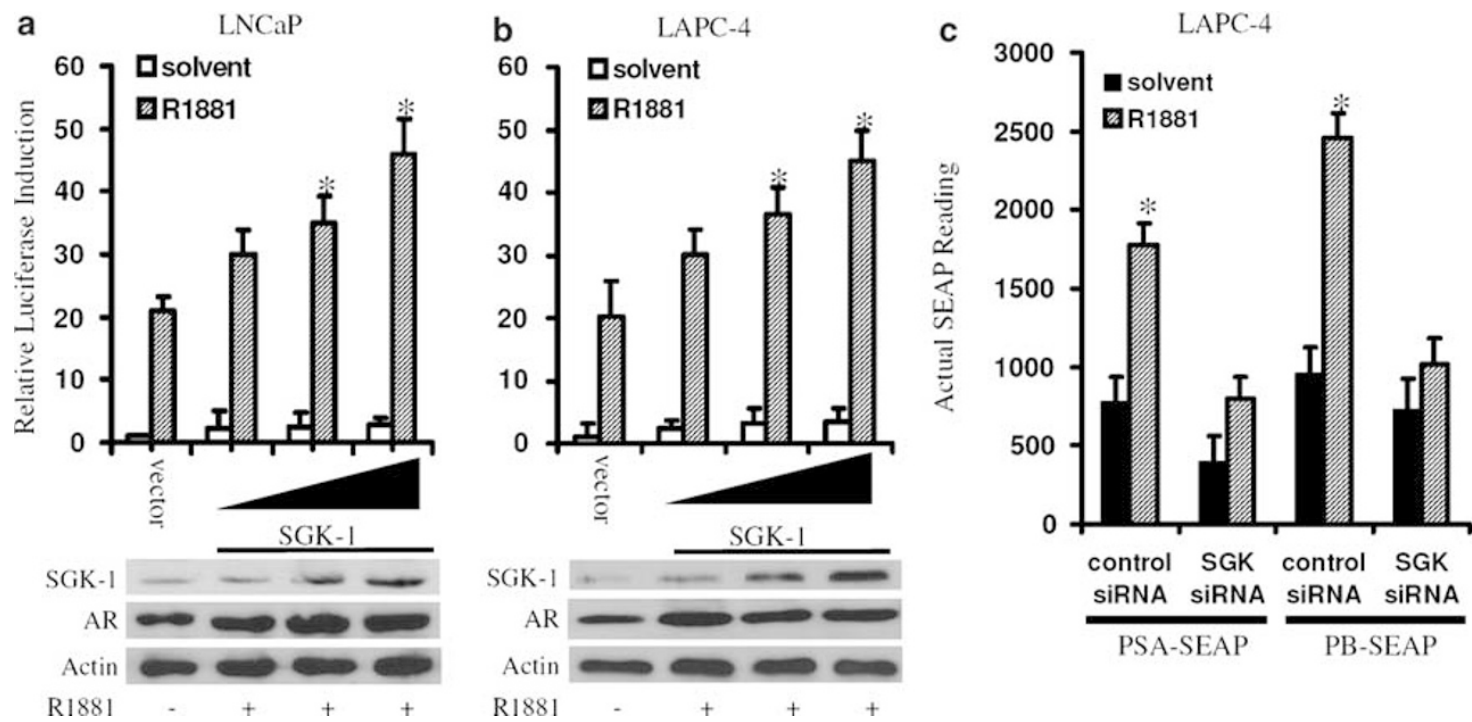

Figure 6 SGK-1 modulates androgen receptor-mediated gene expression. $(\mathbf{a}, \mathbf{b})$ LNCaP or LAPC-4 cells were placed on six-well plates overnight and were transfected with an androgen response gene reporter ARE-LUC (1.0 $\mu$ gwell) together with increasing amounts of SGK-1 expression constructs (pCMV6-5XL-hSGK1) at 0.25, 0.5, 1.0 $\mu \mathrm{g} /$ well. An empty vector (pCMV6-5XL) was used for the control transfection. Cells were serum-starved for $24 \mathrm{~h}$ and then treated with R1881 (1.0 nM) or the solvent in $5 \% \mathrm{cFBS}$ containing media. Luciferase activities were assessed $24 \mathrm{~h}$ later and presented as described earlier. Expression levels of SGK-1 or AR were assessed by immunoblotting, and anti-Actin blot was used as loading control. (c) LAPC-4 cells were transfected with SGK-1 siRNA or the negative control siRNA for 3 days and then transfected again with the SEAP reporter constructs as indicated. After $24 \mathrm{~h}$ serum starvation, cells were stimulated with R1881 or the solvent in $5 \%$ cFBS-containing media. SEAP activities were assessed $24 \mathrm{~h}$ later as described earlier. Data were from three separated experiments and error bar represents S.E. The asterisk indicates a significant difference $(P<0.05, t-$ test) compared to the solvent or the vector controls 
Figure $6 a$ and b, SGK-1 overexpression significantly enhanced R1881-stimulated ARE-LUC activity, in an SGK1 dose-dependent manner. In addition, we also noticed that the basal reporter activities in the absence of R1881 were increased by 2-3 folds in SGK-1-transfected cells, although the absolute luciferase reading was much lower than that in R1881-treated cells. These data suggest that SGK-1 overexpression enhances $A R$ transactivation in both the presence or absence of androgen.

Finally, we tested if SGK-1 is required for AR transactivation. We used two SEAP reporters driven by different promoters derived from the endogenous AR target genes, human psa (PSA-E2/P-SEAP, termed as PSA-SEAP) and rat probasin (ARR ${ }_{2} \mathrm{~PB}-\mathrm{SEAP}$, termed as PB-SEAP), as described previously. ${ }^{33,34}$ LAPC-4 cells were transfected with SGK-1 siRNA for 3 days to eliminate SGK-1 expression. Cells were then transfected again with the reporter constructs. After R1881 treatment for $24 \mathrm{~h}$, cell culture medium was collected for SEAP activity. As shown in Figure $6 c$, compared to the solvent control, R1881 stimulation significantly increased SEAP activities from both reporters in cells transfected with the control siRNA. In SGK-1 siRNA-transfected cells, however, R1881-stimulated SEAP activities were completely blocked. Furthermore, the basal SEAP activity (the solvent control) was also slightly reduced in SGK-1 siRNA-transfected cells compared to the control siRNA-transfected cells. These data suggest a critical role of SGK-1 in androgenstimulated AR transactivation.

\section{Discussion}

It is well known that the AR is essential for cellular survival in prostate cancer cells, ${ }^{8-15}$ but its downstream effectors are not fully listed. In this study, we sought to identify novel downstream mediators responsible for AR-dependent survival. First, we used a genome-wide approach to screen the genes altered after androgen stimulation. SGK-1 was identified as one of the significantly upregulated genes (Table 1), which was supported by a recent report. ${ }^{21} \mathrm{AR}$ dependent SGK-1 expression was then validated at both mRNA and protein levels (Figure 1). After androgen stimulation, the AR was recruited onto the SGK-1 promoter that contains at least two putative motifs for AR binding, which confirms a previous computer-based promoter inspection. ${ }^{20}$ Consistently, androgen activated SGK-1 promoter in an ARdependent manner (Figures 2 and 3). SGK-1 siRNA reduced androgen-mediated death protection from serum starvation (Figures 4 and 5). SGK-1 overexpression not only abrogated AR siRNA-induced apoptosis, but also enhanced AR transactivation in prostate cancer cells (Figure 6); this later event reflects a positive feedback loop of AR-mediated SGK-1 expression and survival, as illustrated in Figure 7. All together, our data demonstrated that SGK-1 is a pivotal factor in ARdependent survival pathway.

As an early response gene, SGK-1 expression is increased by many external or internal stimuli, including steroid hormones, peptide growth factors, cytokines, and cellular physical or genetic stresses (reviewed by Tessier and Woodgett ${ }^{18}$ ). We and others have previously identified a GRE motif (5'-CGGACAaaaTGTTCT-3') at -1159/-1145

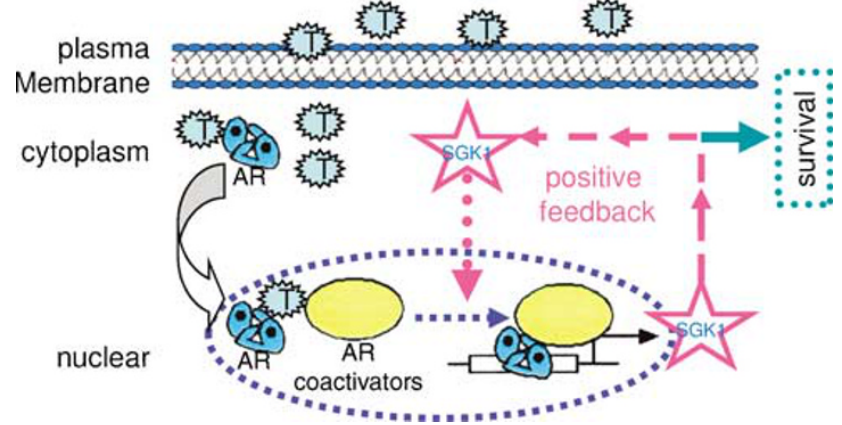

Figure 7 Proposed mechanism for SGK-1 expression in AR-dependent survival. Following androgen stimulation, the androgen receptor (AR) transactivates SGK-1 expression. AR-mediated SGK-1 expression promotes survival and then in turn enhances AR-mediated gene expression, which reflects a positive feedback in AR-dependent survival and gene expression

region in sgk-1 gene promoter, which is responsible for glucocorticoid-induced SGK-1 expression. ${ }^{16,24}$ In this study, we found that the AR also interacted with this region after androgen stimulation. Reporter gene assay showed that the promoter fragment containing this GRE motif was activated by androgen treatment at AR-dependent manner in LNCaP cells that are null of glucocorticoid receptor (GR). ${ }^{35}$ Because the $A R$ and the GR are closely related members in the steroid hormone receptor family, ${ }^{22}$ it is plausible that this GRE (termed as ARE-1) motif is involved in androgen-induced SGK-1 expression.

In addition to the previously identified GRE/ARE-1 motif, a computer-based motif inspection proposed a secondary GRE/ ARE motif in the distal region of -2.5 to $-3.2 \mathrm{~kb}$ in the $s g k$ promoter. ${ }^{19,20}$ In this study, our data convincingly indicate that a functional ARE motif indeed exists within this distal region. The promoter fragment (442-SEAP) derived from this distal region functionally responded to androgen stimulation (Figure $2 \mathrm{~d}$ and $\mathrm{e}$ ) and the AR was recruited onto this region (Figure 3 ). A close examination of the promoter sequence revealed a putative ARE motif (5'-GGCTATcccTGTTCT-3', termed as $A R E-2)$ at the region of -2965 to -2950 related to the transcription site. Although the actual identity of the secondary ARE motif is under further investigation, our data indicate that this distal region containing the ARE-2 motif might act as an enhancer in androgen-induced SGK-1 expression.

Because of its close relationship with Akt, SGK-1 was considered as a major cellular survival factor. A previous report demonstrated that SGK-1, but not Akt, is induced by various environmental stress stimuli and then subsequently protects cell from apoptosis induced by environmental stress. ${ }^{27}$ Similarly, in this study, we determined that SGK-1 but not other sgk isoforms or Akt was upregulated after androgen stimulation. Our data also demonstrated that SGK1 is critical for androgen-mediated survival in prostate cancer cells because eliminating SGK-1 expression abrogated androgen-induced death protection.

Early studies demonstrated that SGK-1 exerts its survival function via inactivating proapoptotic transcription factor FKHRL1. ${ }^{26,27,29,36}$ A recent study discovered a novel mechanism that SGK-1 promotes NF- $\kappa$ B survival pathway by activating $\operatorname{IKK} \beta .^{28}$ In this study, we found that in $A R$ 
siRNA-induced apoptosis, $1 \kappa \mathrm{B}-\alpha$ phosphorylation was largely reduced in association with SGK-1 downregulation (Figure 5d). Conversely, SGK-1 overexpression reduced AR siRNA-induced apoptosis, which was associated with increased $\mid \kappa \mathrm{B}-\alpha$ phosphorylation. These data indicate that SGK-1 downregulation due to AR silencing resulted in reduction of $\mathrm{IKK} \beta$ activity, leading to loss of $\mathrm{I}_{\kappa} \mathrm{B}-\alpha$ phosphorylation and eventually apoptotic cell death.

PI3K is a major factor involved in multiple cellular processes; both Akt and SGK-1 are PI3K downstream targets. ${ }^{18}$ We previously showed that PI3K activity is required for androgen-induced AR transactivation and gene expression, and that Akt is dispensable for AR transactivation. ${ }^{33}$ In this study, we found that SGK-1 is required for AR-dependent cellular survival and gene expression, indicating that SGK-1 might be one of the PI3K downstream effectors in androgen signaling. Although both Akt and SGK-1 use a similar phosphorylation preference of $\mathrm{RxR} x \mathrm{xS} / \mathrm{T}$ on their substrates, some distinctions were recently reported in terms of their substrate specificity requirements. ${ }^{37}$ This substrate specificity might be the reason for the requirement of SGK-1 but not Akt in AR signaling, which will be worthwhile for further investigation.

In conclusion, we demonstrated in this study that protein kinase SGK-1 is an androgen-inducible gene, and is required for AR-dependent cellular survival and gene expression in prostate cancer cells. Further study is desirable to understand the mechanisms for SGK-1 regulation of AR signaling.

\section{Materials and Methods}

Cell culture and reagents. The human prostate cancer LNCaP and LAPC- 4 cells and their culture conditions were described previously. ${ }^{12,33,38-40}$ LAPC-4/ SGK1 and LNCaP/SGK1 sublines were established by stably infecting the parental cells with a retrovirus vector (pMSCV-puro-SGK1) ${ }^{29}$ bearing the rat sgk1 gene. The control subline LAPC-4/Puro and LNCaP/Puro were established when an empty vector was used. The stable clones were selected in puromycin-containing media. A mammalian expression vector harboring human sgk1 cDNA (pCMV6-XL5-hSGK1, OriGene Technologies Inc., Rockville, MD) was used for transient transfection. Antibodies against human AR, SGK-1, Akt, $\beta$-Actin as well as secondary antibodies were purchased from Santa Cruz Biotech (Santa Cruz, CA). Antibodies for human caspase-3, PARP, phospho-specific $l_{\kappa} \mathrm{B}-\alpha$, and plain $\left.\right|_{\kappa} \mathrm{B}-\alpha$ were obtained from Cell Signaling (Beverly, MA). CFBS and regular FBS were obtained from Atlanta Biologicals (Norcross, GA). The synthetic androgen R1881 was purchased from Perkin-Elmer (Wellesley, MA). Other reagents were supplied by Sigma (Saint Louis, MO).

RNA extraction, RT-PCR, real-time RT-PCR, and CDNA microarray. Total RNA was prepared using TriZo ${ }^{\mathrm{TM}}$ reagent (Invitrogen Co., Carlsbad, CA). To assess mRNA expression, RT-PCR was carried out using a RETROscript ${ }^{\mathrm{TM}}$ kit from Ambion Inc. as per the manufacturer's manual (Austin, TX). The primers and PCR conditions were described as follow: for human sgk1 gene (forward $5^{\prime}$ atgacggtgaaaactgaggc- $3^{\prime}$; backward $5^{\prime}$-gaggtgtcttgcggaatttg-3'); for human akt1 gene (forward $5^{\prime}$-gtgaaggagggctggctgcacaaacgag-3'; backward $5^{\prime}$ gggtgagcctgatcggaagtccatc- $3^{\prime}$ ). For human PSA gene and $28 \mathrm{~S}$ ribozyme RNA, the primer sequences were described in our previous publication. ${ }^{12}$ The primers were synthesized by IDT Inc. (Coralville, IA). The amplification profile was as follows: $95^{\circ} \mathrm{C}$ for $30 \mathrm{~s}, 56^{\circ} \mathrm{C}$ for $30 \mathrm{~s}$, and $72 \mathrm{C}$ for 1 min running in a total of 25 cycles. After 25 amplification cycles, the expected PCR products were size fractionated onto a $2 \%$ agarose gel and stained with ethidium bromide.

The real-time PCR was performed using the Applied Biosystems 7500 system and the TaqMan ${ }^{\mathrm{TM}}$ Gene Expression Assay kits (Applied Biosystems, Foster City, $\mathrm{CA})$. Reaction mixture consisted of diluted CDNA sample solution $(1.0 \mu \mathrm{l}$ for both SGK-1 and $\beta$-actin), $10 \mu \mathrm{l}$ of TaqMan ${ }^{\mathrm{TM}}$ universal PCR master mixture, $1.0 \mu \mathrm{l}$ of FAM conjugated primer mixtures (Catalog\# 4331182). Final volume was $20 \mu$ l with distilled water. Denaturizing at $95^{\circ} \mathrm{C}$ for $15 \mathrm{~s}$, and annealing and extension at $60^{\circ} \mathrm{C}$ for $1 \mathrm{~min}$ was then performed. The denaturizing/annealing cycle was repeated 40 cycles. Relative value of $s g k 1$ gene expression compared to the control was normalized against $\beta$-actin in each sample.

LNCaP cells were used for cDNA-based microarray analysis of androgenstimulated gene expression. After serum starvation for $48 \mathrm{~h}$, cells were treated with $\mathrm{R} 1881(1.0 \mathrm{nM})$ in serum-free media whereas the control cells received the solvent in the same media. At the end of treatment, total cellular RNAs were extracted using a Trizol-based protocol as described earlier. Genechip ${ }^{\circledR}$ arrays (Affymetrix Inc., Santa Clara, CA) was utilized and the protocol was described in our recent publication. ${ }^{38}$ Briefly, $10 \mu \mathrm{g}$ of total RNAs were used for the first-strand cDNA synthesis with the SuperscriptChoice system (Invitrogen). The in vitro-transcription labeling was conducted using the EnzoBio array high-yield RNA transcript labeling kit (Affymetrix). Genechips were hybridized for $16 \mathrm{~h}$ at $45^{\circ} \mathrm{C}$ and 60 r.p.m. in a Genechip hybridization oven. After washing, the signal intensity was detected using an Agilent Gene Array scanner. Microarray data were analyzed using GeneSpring software (Agilent Technologies, Inc.).

Western blot analysis. For protein expression analysis, western blot was performed as described previously. ${ }^{12,33,38-40}$ Briefly, after treatment, cells were pelleted and lysed in a buffer containing protease inhibitors (Half ${ }^{\mathrm{TM}}$ Protease Inhibitor Cocktail Kit, PIERCE, Rockford, IL), separated on SDS-PAGE gels and transferred to PVDF membrane (BIO-RAD, Hercules, CA). Membranes were blocked in a Tris-buffered solution plus $0.1 \%$ Tween-20 (TBS-T) solution with $5 \%$ nonfat dry milk and incubated with primary antibodies overnight at $4{ }^{\circ} \mathrm{C}$. Immunoreactive signals were detected by horseradish peroxidase-conjugated secondary antibodies (Santa Cruz Biotech, Santa Cruz, CA) followed by SuperSingnal $^{\circledR}$ WestFemto Maximum Sensitivity Substrate (PIERCE Biotechnology, Rockford, IL).

ChIP analysis. The ChIP assay was performed using a ChIP-IT ${ }^{\mathrm{TM}}$ assay kit (Active Motif, Carlsbad, CA) as described in our recent publication. ${ }^{38}$ Cells were maintained in 15-cm dishes and serum-starved for $24 \mathrm{~h}$. After pretreatment with anti-androgen Bicalutamide (Casodex, CSDX), cells were stimulated with R1881. Polyclonal antibodies against AR (Clone C815), RNA polymerase II, GAPDH, and normal rabbit serum were obtained from Santa Cruz Biotech (Santa Cruze, CA). Proper control experiments were performed as described in our recent publication. $^{38}$ The primers for the PCR reactions were: fragment $-3382 /-2940$ (442a $5^{\prime}$-gataagtttagccgaccatccg tctc-3'; 442b $5^{\prime}$-gagccagagacggctatccctgttc-3'); fragment $-1548 /-1130$ (418a $5^{\prime}$-caccggcatcgctg ttctgcagag-3'; 418b $5^{\prime}$-ctgagaac attttgtccgttccgc- $\left.3^{\prime}\right)$; fragment $-852 /-290$ (562a $5^{\prime}$-ctgagtcacggcg gctccttcaag- $3^{\prime}$; $562 \mathrm{~b} 5^{\prime}$-ctctctcatttattccgccgccggag- $\left.3^{\prime}\right)$. The primer binding sites were illustrated in Figure $2 \mathrm{a}$.The PCR products were separated on $1 \%$ agarose gels and stained with ethidium bromide for visualization.

Reporter plasmid construction and reporter gene assays. The pGL3-SGK1/F4R4-Luc reporter construct was described previously, ${ }^{24}$ and the internal control reporter pCMV-SEAP, expressing the secreted alkaline phosphatase (SEAP) under the control of the cytomegalovirus (CMV) promoter and the reporter assays were performed as described previously. ${ }^{12,33,38,40}$ To generate different promoter fragments (Figure 2a) from sgk1 promoter, the PCR fragments amplified from $\mathrm{LNCaP}$ cell genomic DNA using the primers described in ChIP assays were subcloned into the pCR2.1 vector (Invitrogen, Carlsbad, CA) and the accuracy of the inserts were confirmed by direct sequencing. Then, the individual fragment was released from the pCR2.1 vector using Hindll/Xbal digestion and directionally ligated into pSH1-ARR2PB-SEAP vector as described previously, ${ }^{33}$ so as to generate $s g k 1$ fragment-driven SEAP gene reporters by replacing the probasin promoter. The ARE-LUC reporter was described previously. ${ }^{41}$

The reporter gene assay was performed as described previously. ${ }^{12,33,38,40}$ Briefly, cells plated in six-well tissue culture plates were transfected with reporter constructs using the Cytofectene ${ }^{\mathrm{TM}}$ reagent (BioRad) according to the manufacturer's protocol. After $24 \mathrm{~h}$, cells were serum-starved for another $24 \mathrm{~h}$, and then pretreated with anti-androgen CSDX or the solvent. Cell were treated with the solvent or stimulated with R1881 in 5\% cFBS. Culture supernatants and cell lysates were collected for reporter assays. The luciferase reporter activity of each sample was normalized against the corresponding SEAP activity and protein concentration prior to calculation of the relative fold induction compared to the controls. 
SiRNA transfection, cell viability, and clonogenic assay. Predefined siRNA against human $s g k 1$ gene and a negative control siRNA with scramble sequence were purchased from Santa Cruz Biotechnology and transfected with OligoFectamine ${ }^{\mathrm{TM}}$ (Invitrogen) according to the manufacturer's manual. For cell viability experiments, cells were seeded at the density of $2 \times 10^{4}$ cells in 12-well plates in duplicates and allowed to attachment overnight. One week after siRNA transfection, cells were subjected to viability assay. Cells were harvested at the end of experiments and the number of viable cells was counted using a hemocytometer after staining with trypan blue as described previously. ${ }^{12,40}$

For the clonogenic assay, $1.0 \times 10^{3}$ cells were seeded in a $35-\mathrm{mm}$ dish and transfected with SGK-1 siRNA or the control siRNA. The cultures were monitored daily for colony formation in a $2 \%$ cFBS-containing media. One week later, the cultures were washed with phosphate-buffered saline (PBS), fixed, and stained as described previously. ${ }^{40}$ The colonies were counted and then photographed using a digital camera.

AAV production and infection. To silence AR gene expression, a hairpinstructured expression vector was created using pSilencer-U6 1.0 plasmid vector (Ambion Inc., Austin, TX) based on our previously published AR siRNA sequence. ${ }^{12}$ The oligonucleotide sequences were as follow: sense, $5^{\prime}$-GAAGGCCAGTTG TATGGACttcaagagaGTCCATACAA CTGGCCTTC ttttt- $3^{\prime}$ and antisense, $5^{\prime}$-attaa aaaGAAGGC CAGTTGTATGGACtctcttgaaGTCCATACAACT GGCCTTC $g g c c-3^{\prime}$. Annealed DNA fragment was subcloned into Apal/EcoRl sites of pSilencer-U6 vector, and the resultant vector named as pSilencer-U6-ARHP8. To create an AAV for ARHP8 siRNA expression, the AAV Helper-free system (Stratagene, La Jolla, $\mathrm{CA}$ ) was utilized. The ARHP8 expression cassette was released by Xbal digestion and subcloned into pCMV-MCS from the AAV system. Then, this fragment was moved into $\mathrm{pAAV}$-IRES-hGFP by EcoRI/Xhol digestion/ligation process to generate AAV-ARHP8 viral-producing vector. The PAAV-IRES-hGFP construct was used to produce an empty AAV virus (AAV-GFP). These AAV particles were packaged and purified by Applied Viromics LLC (Fremont, CA) at a titer of $10^{12}$ viral particle per ml.

Statistical analysis. All experiments were repeated two or three times. RT-PCR results are presented from a representative experiment. The mean and standard error (S.E.) from multiple experiments for real-time RT-PCR, cell viability, or reporter gene assay are shown. The significant differences between groups were analyzed as described previously ${ }^{12,33,38-40}$ using the SPSS computer software (SPSS Inc., Chicago, IL).

Acknowledgements. We are grateful for Dr. Jie Du (Department of Medicine Baylor College of Medicine, Houston, TX) for the generous gift of the retrovirus expressing rat SGK-1. We Thank Mrs. Donna Barnes for her excellent secretaria assistance and Dr. Stan Svojanovsky for technical assistance in CDNA microarray data analysis, which was performed in KUMC K-INBRE Bioinformatics Core facility under the financial support by an $\mathrm{NIH}$ grant P20RR016475. This study was supported by the KU William L. Valk Endowment, and grants from Kansas Mason's Foundation, KUMC Lied Foundation and the Department of Defense Idea Development Award (W81XWH-04-0214) to Dr. Benyi Li.

1. Heinlein CA, Chang C. Androgen receptor in prostate cancer. Endocr Rev 2004; 25 276-308.

2. Chang CS, Kokontis J, Liao ST. Molecular cloning of human and rat complementary DNA encoding androgen receptors. Science 1988; 240: 324-326.

3. Roy AK, Tyagi RK, Song CS, Lavrovsky Y, Ahn SC, Oh TS et al. Androgen receptor: structural domains and functional dynamics after ligand-receptor interaction. Ann $N$ Y Acad Sci 2001; 949: 44-57.

4. Kyprianou N, Isaacs JT. Activation of programmed cell death in the rat ventral prostate after castration. Endocrinology 1988; 122: 552-562.

5. Lee EC, Zhan P, Schallhom R, Packman K, Tenniswood M. Antiandrogen-induced cell death in LNCaP human prostate cancer cells. Cell Death Differ 2003; 10: 761-771.

6. Huggins $\mathrm{C}$, Hodges CV. Studies on prostatic cancer: the effects of castration, of estrogen and of androgen injection on serum phosphatases in metastatic carcinoma of the prostate. Cancer Res 1941; 1: 293-297.

7. Scher HI, Sawyers CL. Biology of progressive, castration-resistant prostate cancer: directed therapies targeting the androgen-receptor signaling axis. J Clin Oncol 2005; 23: 8253-8261.

8. Chen CD, Welsbie DS, Tran C, Baek SH, Chen R, Vessella R et al. Molecular determinants of resistance to antiandrogen therapy. Nat Med 2004; 10: 33-39.
9. Zhang $\mathrm{L}$, Johnson $\mathrm{M}$, Le $\mathrm{KH}$, Sato $\mathrm{M}$, llagan $\mathrm{R}$, lyer $\mathrm{M}$ et al. Interrogating androgen receptor function in recurrent prostate cancer. Cancer Res 2003; 63: 4552-4560.

10. Zegarra-Moro OL, Schmidt LJ, Huang H, Tindall DJ. Disruption of androgen receptor function inhibits proliferation of androgen-refractory prostate cancer cells. Cancer Res 2002; 62: 1008-1013.

11. Stanbrough M, Leav I, Kwan PW, Bubley GJ, Balk SP. Prostatic intraepithelial neoplasia in mice expressing an androgen receptor transgene in prostate epithelium. Proc Natl Acad Sci USA 2001; 98: 10823-10828.

12. Liao X, Tang S, Thrasher JB, Griebling TL, Li B. Small-interfering RNA-induced androgen receptor silencing leads to apoptotic cell death in prostate cancer. Mol Cancer Ther 2005; 4: $505-515$

13. Yang $Q$, Fung KM, Day WV, Kropp BP, Lin HK. Androgen receptor signaling is required for androgen-sensitive human prostate cancer cell proliferation and survival. Cancer Cell Int 2005; 5 : 8.

14. Haag P, Bektic J, Bartsch G, Klocker H, Eder IE. Androgen receptor down regulation by small interference RNA induces cell growth inhibition in androgen sensitive as well as in androgen independent prostate cancer cells. J Steroid Biochem Mol Biol 2005; 96: 251-258.

15. Cheng H, Snoek R, Ghaidi F, Cox ME, Rennie PS. Short hairpin RNA knockdown of the androgen receptor attenuates ligand-independent activation and delays tumor progression. Cancer Res 2006; 66: 10613-10620.

16. Webster MK, Goya L, Ge Y, Maiyar AC, Firestone GL. Characterization of sgk, a novel member of the serine/threonine protein kinase gene family which is transcriptionally induced by glucocorticoids and serum. Mol Cell Biol 1993; 13: 2031-2040.

17. Kobayashi T, Deak M, Morrice N, Cohen P. Characterization of the structure and regulation of two novel isoforms of serum- and glucocorticoid-induced protein kinase. Biochem $J$ 1999; 344 (Part 1): 189-197.

18. Tessier M, Woodgett JR. Serum and glucocorticoid-regulated protein kinases: variations on a theme. J Cell Biochem 2006; 98: 1391-1407.

19. Loffing J, Flores SY, Staub O. Sgk kinases and their role in epithelial transport. Annu Rev Physiol 2006; 68: 461-490.

20. Firestone GL, Giampaolo JR, O'Keeffe BA. Stimulus-dependent regulation of serum and glucocorticoid inducible protein kinase (SGK) transcription, subcellular localization and enzymatic activity. Cell Physiol Biochem 2003; 13: 1-12.

21. Xu Y, Chen SY, Ross KN, Balk SP. Androgens induce prostate cancer cell proliferation through mammalian target of rapamycin activation and post-transcriptional increases in cyclin D proteins. Cancer Res 2006; 66: 7783-7792.

22. Laudet V, Hanni C, Coll J, Catzeflis F, Stehelin D. Evolution of the nuclear receptor gene superfamily. EMBO J 1992; 11: 1003-1013.

23. Claessens F, Verrijdt G, Schoenmakers E, Haelens A, Peeters B, Verhoeven G et al. Selective DNA binding by the androgen receptor as a mechanism for hormone-specific gene regulation. J Steroid Biochem Mol Biol 2001; 76: 23-30.

24. Itani OA, Liu KZ, Cornish KL, Campbell JR, Thomas CP. Glucocorticoids stimulate human sgk1 gene expression by activation of a GRE in its $5^{\prime}$-flanking region. Am J Physiol Endocrinol Metab 2002; 283: E971-E979.

25. Maiyar AC, Phu PT, Huang AJ, Firestone GL. Repression of glucocorticoid receptor transactivation and DNA binding of a glucocorticoid response element within the serum/ glucocorticoid-inducible protein kinase (sgk) gene promoter by the p53 tumor suppressor protein. Mol Endocrinol 1997; 11: 312-329.

26. Mikosz CA, Brickley DR, Sharkey MS, Moran TW, Conzen SD. Glucocorticoid receptormediated protection from apoptosis is associated with induction of the serine/threonine survival kinase gene, sgk-1. J Biol Chem 2001; 276: 16649-16654.

27. Leong ML, Maiyar AC, Kim B, O'Keeffe BA, Firestone GL. Expression of the serum- and glucocorticoid-inducible protein kinase, Sgk, is a cell survival response to multiple types of environmental stress stimuli in mammary epithelial cells. J Biol Chem 2003; 278: 5871-5882.

28. Zhang L, Cui R, Cheng X, Du J. Antiapoptotic effect of serum and glucocorticoid-inducible protein kinase is mediated by novel mechanism activating I\{kappa\}B kinase. Cancer Res 2005; 65: 457-464.

29. Brunet A, Park J, Tran H, Hu LS, Hemmings BA, Greenberg ME. Protein kinase SGK mediates survival signals by phosphorylating the forkhead transcription factor FKHRL1 (FOXO3a). Mol Cell Biol 2001; 21: 952-965.

30. Traenckner EB, Pahl HL, Henkel T, Schmidt KN, Wilk S, Baeuerle PA. Phosphorylation of human I kappa B-alpha on serines 32 and 36 controls I kappa B-alpha proteolysis and NF-kappa B activation in response to diverse stimuli. EMBO J 1995; 14: 2876-2883.

31. Suh J, Rabson AB. NF-kappaB activation in human prostate cancer: important mediator or epiphenomenon? J Cell Biochem 2004; 91: 100-117.

32. Lang F, Bohmer C, Palmada M, Seebohm G, Strutz-Seebohm N, Vallon V. (Patho)physiological significance of the serum- and glucocorticoid-inducible kinase isoforms. Physiol Rev 2006; 86: 1151-1178.

33. Liao X, Thrasher JB, Holzbeierlein J, Stanley S, Li B. Glycogen synthase kinase-3beta activity is required for androgen-stimulated gene expression in prostate cancer. Endocrinology 2004; 145: 2941-2949.

34. Xie X, Zhao X, Liu Y, Zhang J, Matusik RJ, Slawin KM et al. Adenovirus-mediated tissuetargeted expression of a caspase-9-based artificial death switch for the treatment of prostate cancer. Cancer Res 2001; 61: 6795-6804.

35. Nishimura K, Nonomura N, Satoh E, Harada Y, Nakayama M, Tokizane T et al. Potential mechanism for the effects of dexamethasone on growth of androgen-independent prostate cancer. J Natl Cancer Inst 2001; 93: 1739-1746. 
36. You H, Jang Y, You-Ten Al, Okada H, Liepa J, Wakeham A et al. p53-dependent inhibition of FKHRL1 in response to DNA damage through protein kinase SGK1. Proc Natl Acad Sci USA 2004; 101: 14057-14062.

37. Murray JT, Cummings LA, Bloomberg GB, Cohen P. Identification of different specificity requirements between SGK1 and PKBalpha. FEBS Lett 2005; 579: 991-994.

38. Sun A, Shanmugam I, Song J, Terranova PF, Thrasher JB, Li B. Lithium suppresses cell proliferation by interrupting E2F-DNA interaction and subsequently reducing S-phase gene expression in prostate cancer. Prostate 2007; 67: 976-988.
39. Li B, Sun A, Youn H, Hong Y, Terranova PF, Thrasher JB et al. Conditional Akt activation promotes androgen-independent progression of prostate cancer. Carcinogenesis 2007; 28: $572-583$.

40. Liao X, Zhang L, Thrasher JB, Du J, Li B. Glycogen synthase kinase-3beta suppression eliminates tumor necrosis factor-related apoptosis-inducing ligand resistance in prostate cancer. Mol Cancer Ther 2003; 2: 1215-1222.

41. Lee SO, Lou W, Hou M, Onate SA, Gao AC. Interleukin-4 enhances prostate-specific antigen expression by activation of the androgen receptor and Akt pathway. Oncogene 2003; 22: 7981-7988. 